\title{
Application of Hybrid Functions for Solving Duffing-Harmonic Oscillator
}

\author{
Mohammad Heydari, ${ }^{1}$ Ghasem Brid Loghmani, \\ Seyed Mohammad. Hosseini, ${ }^{2}$ and Seyed Mehdi Karbassi ${ }^{3}$ \\ ${ }^{1}$ Department of Mathematics, Yazd University, Yazd, Iran \\ ${ }^{2}$ Department of Mathematics, Islamic Azad University, Shahrekord Branch, Shahrekord, Iran \\ ${ }^{3}$ Department of Mathematics, Islamic Azad University, Yazd Branch, Yazd, Iran
}

Correspondence should be addressed to Mohammad Heydari; m.heydari85@gmail.com

Received 9 April 2014; Revised 26 July 2014; Accepted 28 July 2014; Published 14 August 2014

Academic Editor: Athanassios G. Bratsos

Copyright (C) 2014 Mohammad Heydari et al. This is an open access article distributed under the Creative Commons Attribution License, which permits unrestricted use, distribution, and reproduction in any medium, provided the original work is properly cited.

\begin{abstract}
A numerical method for finding the solution of Duffing-harmonic oscillator is proposed. The approach is based on hybrid functions approximation. The properties of hybrid functions that consist of block-pulse and Chebyshev cardinal functions are discussed. The associated operational matrices of integration and product are then utilized to reduce the solution of a strongly nonlinear oscillator to the solution of a system of algebraic equations. The method is easy to implement and computationally very attractive. The results are compared with the exact solution and results from several recently published methods, and the comparisons showed proper accuracy of this method.
\end{abstract}

\section{Introduction}

Most phenomena in our world are essentially nonlinear and are described by nonlinear ordinary differential equations. Nonlinear oscillation in mechanics, physics, and applied mathematics has been a topic of intensive research for many years. Difficulty of solving the nonlinear problems or getting an analytic solution leads one to use numerical methods. Several methods have been used to find approximate solutions to these nonlinear problems. Some of these well-known methods are harmonic balance method [1], multiple scales method [2], Krylov-Bogoliubov-Mitropolsky method [3, 4], modified Lindstedt-Poincare method [5], linearized perturbation method [6], energy balance method [7], iteration perturbation method [8], bookkeeping parameter perturbation method [9], amplitude frequency formulation [10], maximum approach [11], Mickens iteration procedure [12], rational harmonic balance method [13], Adomian decomposition method [14], variational iteration method [15], modified variational iteration method [16, 17], homotopy perturbation method [18], modified differential transform method [19], and modified homotopy perturbation method [20].

Recently, hybrid functions have been applied extensively for solving differential equations or systems and proved to be a useful mathematical tool. The pioneering work in the solution of linear systems with inequality constraints via hybrid of block-pulse functions and Legendre polynomials was led in [21] that first derived an operational matrix for the integrals of the hybrid function vector. Razzaghi and Marzban in [22] the variational problems are solved using hybrid of blockpulse and Chebyshev functions. Razzaghi and Marzban [23] applied the hybrid of block-pulse and Chebyshev functions to find approximate solution of systems with delays in state and control. Solution of time-varying delay systems is approximated using hybrid of block-pulse functions and Legendre polynomials in [24]. Maleknejad and Tavassoli Kajani in [25] introduced a Galerkin method based on hybrid Legendre and block-pulse functions on interval $[0,1)$ to solve the linear integrodifferential equation system. Razzaghi and Marzban 
in [26], a direct method for solving multidelay systems using hybrid of block-pulse functions and Taylor series is presented. Marzban et al. [27] implemented hybrid of blockpulse functions and Lagrange-interpolating polynomials to find approximate solution of Volterra's population model. The Lane-Emden type equations are solved in [28] using hybrid functions of block-pulse and Lagrange-interpolating polynomials. The hybrid of block-pulse functions and Taylor series is employed in [29] to solve the linear quadratic optimal control with delay systems. Application of hybrid of blockpulse functions and Lagrange polynomials for solving the nonlinear mixed Volterra-Fredholm-Hammerstein integral equations is investigated in [30].

In this study, we consider the following nonlinear Duffing-harmonic oscillation [31-35]:

$$
u^{\prime \prime}+\frac{u^{3}}{1+u^{2}}=0, \quad u(0)=A, \quad u^{\prime}(0)=0,
$$

where is an example of conservative nonlinear oscillatory systems having a rational form for the restoring force. Note that, for small values of $u,(1)$ is that of a Duffing-type nonlinear oscillator; that is,

$$
u^{\prime \prime}+u^{3} \simeq 0
$$

while for large values of $u$ the equation approximates that of a linear harmonic oscillator; that is,

$$
u^{\prime \prime}+u \simeq 0
$$

Hence, (1) is called the Duffing-harmonic oscillator [31]. The system will oscillate between symmetric bounds $[-A, A]$, and the frequency and corresponding periodic solution of the nonlinear oscillator are dependent on the amplitude $A$ [33].

In this paper, we introduce an alternative numerical method to solve Duffing-harmonic oscillator. The method consists of reducing this equation to a set of algebraic equations by first expanding the candidate function as a hybrid function with unknown coefficients. These hybrid functions, which consist of block-pulse functions plus Chebyshev cardinal functions, are first introduced. The operational matrices of integration and product are given. These matrices are then used to evaluate the coefficients of the hybrid function for the solution of strongly nonlinear oscillators.

The outline of this paper is as follows. In Section 2, the basic properties of hybrid block-pulse functions and Chebyshev cardinal functions required for subsequent development are described. In Section 3, we apply the proposed numerical method to the Duffing-harmonic oscillation. Results and comparisons with existing methods in the literature are presented in Section 4 and finally conclusions are drawn in Section 5.

\section{Properties of Hybrid Functions}

Marzban et al. in $[27,29,30]$ used the hybrid of block-pulse functions and Lagrange-interpolating polynomials based on zeros of the Legendre polynomials. But no explicit formulas are known for the zeros of the Legendre polynomials. In this study we used Chebyshev cardinal functions which are special cases of Lagrange-interpolating polynomials based on zeros of the Chebyshev polynomials of the first kind to overcome this problem. In this paper, we present the properties of hybrid functions which consist of block-pulse functions plus Chebyshev cardinal functions similar to [27, $29,30]$. The hybrid functions are first introduced, and the operational matrices of integration and product are then derived.

2.1. Hybrid Functions of Block-Pulse and Chebyshev Cardinal Functions. Hybrid functions $\theta_{n, m}(t), n=1,2, \ldots N, m=$ $0,1, \ldots, M-1$, are defined on the interval $\left[0, t_{f}\right)$ as

$$
\begin{aligned}
& \theta_{n, m}(t) \\
& \quad= \begin{cases}C_{m}\left(\frac{2 N}{t_{f}} t-2 n+1\right), & t \in\left[\left(\frac{n-1}{N}\right) t_{f}, \frac{n}{N} t_{f}\right), \\
0, & \text { otherwise, }\end{cases}
\end{aligned}
$$

where $n$ and $m$ are the order of block-pulse functions and Chebyshev cardinal functions, respectively. Here, $C_{m}(t)$ are defined as [36-38]

$$
C_{m}(t)=\frac{T_{M}(t)}{T_{M, t}\left(t_{m}\right)\left(t-t_{m}\right)}, \quad m=0,1, \ldots, M-1,
$$

where $T_{M}(t)$ is the first kind Chebyshev polynomial of order $M$ in $[-1,1]$ defined by

$$
T_{M}(t)=\cos (M \arccos (t)),
$$

subscript $t$ denotes $t$-differentiation, and $t_{m}, m=0,1, \ldots$, $M-1$, are the zeros of $T_{M}(t)$ defined by $\left.\cos ((2 m+1) \pi) / 2 M\right)$, $m=0,1, \ldots, M-1$, with the Kronecker property

$$
C_{m}\left(t_{i}\right)=\delta_{m i}= \begin{cases}1, & \text { if } i=m, \\ 0, & \text { if } i \neq m,\end{cases}
$$

where $\delta_{m i}$ is the Kronecker delta function.

2.2. Function Approximation. A function $f(t)$, defined over the interval $\left[0, t_{f}\right)$, may be expanded as

$$
f(t)=\sum_{n=1}^{\infty} \sum_{m=0}^{\infty} c_{n m} \theta_{n m}(t)
$$

If the infinite series in (8) is truncated, then (8) can be expressed as

$$
f(t) \simeq \sum_{n=1}^{N} \sum_{m=0}^{M-1} c_{n m} \theta_{n m}(t)=C^{T} \Theta(t),
$$

where

$$
\begin{gathered}
C=\left[c_{10}, \ldots, c_{1 M-1}, c_{20}, \ldots, c_{2 M-1}, \ldots, c_{N 0}, \ldots, c_{N M-1}\right]^{T}, \\
\Theta(t)=\left[\theta_{10}(t), \ldots, \theta_{1 M-1}(t), \theta_{20}(t), \ldots,\right. \\
\left.\theta_{2 M-1}(t), \ldots, \theta_{N 0}(t), \ldots, \theta_{N M-1}(t)\right]^{T} .
\end{gathered}
$$


In (10) and (11), $c_{n m}, n=1,2, \ldots, N, m=0,1, \ldots, M-1$, are the expansion coefficients of the function $f(t)$ in the $n$th subinterval $\left[((n-1) / N) t_{f},(n / N) t_{f}\right)$ and $\theta_{n m}(t), n=$ $1,2, \ldots, N, m=0,1, \ldots, M-1$, are defined as in (4). With the aid of (7), the coefficients $c_{n m}$ can be obtained as

$$
c_{n m}=f\left(\frac{t_{f}}{2 N}\left(t_{m}+2 n-1\right)\right) \text {. }
$$

2.3. The Operational Matrix of Integration. In this section, the operational matrix of integration is derived. The integration of the vector $\Theta(t)$ defined in (11) can be approximated as

$$
\int_{0}^{t} \Theta(s) d s \simeq P \Theta(t),
$$

where $P$ is the $M N \times M N$ operational matrix of integration for Chebyshev cardinal functions. The matrix $P$ can be obtained by the following process. Let

$$
\begin{aligned}
\int_{0}^{t} \Theta(s) d s \\
=\left[\int_{0}^{t} \theta_{10}(s) d s, \ldots, \int_{0}^{t} \theta_{1 M-1}(s) d s, \ldots,\right. \\
\left.\quad \int_{0}^{t} \theta_{N 0}(s) d s, \ldots, \int_{0}^{t} \theta_{N M-1}(s) d s\right]^{T} .
\end{aligned}
$$

Using (9), any function $\int_{0}^{t} \theta_{l k}(s) d s, l=1,2, \ldots, N, k=$ $0,1, \ldots, M-1$, can be approximated as

$$
\int_{0}^{t} \theta_{l k}(s) d s \simeq \sum_{n=1}^{N} \sum_{m=0}^{M-1} c_{n m} \theta_{n m}(t) .
$$

From (12) we can get

$$
c_{n m}=\int_{0}^{\left(t_{f} / 2 N\right)\left(t_{m}+2 n-1\right)} \theta_{l k}(s) d s .
$$

With the aid of (4), we consider the following cases.

Case 1. If $n<l$, then $\left(t_{f} / 2 N\right)\left(t_{m}+2 n-1\right)<((l-1) / N) t_{f}$ and we obtain $c_{n m}=0$.

Case 2. If $n=l$, then $((l-1) / N) t_{f}<\left(t_{f} / 2 N\right)\left(t_{m}+2 n-1\right)<$ $(l / N) t_{f}$ and we obtain

$$
\begin{aligned}
c_{n m} & \\
& =\int_{((l-1) / N) t_{f}}^{\left(t_{f} / 2 N\right)\left(t_{m}+2 n-1\right)} C_{k}\left(\frac{2 N}{t_{f}} s-2 l+1\right) d s \\
& =\frac{t_{f}}{2 N} \int_{-1}^{t_{m}} C_{k}(v) d v .
\end{aligned}
$$

Case 3. If $n>l$, then $(l / N) t_{f}<\left(t_{f} / 2 N\right)\left(t_{m}+2 n-1\right)$ and we obtain

$$
\begin{gathered}
c_{n m} \\
=\int_{((l-1) / N) t_{f}}^{(l / N) t_{f}} C_{k}\left(\frac{2 N}{t_{f}} s-2 l+1\right) d s=\frac{t_{f}}{2 N} \int_{-1}^{1} C_{k}(v) d v .
\end{gathered}
$$

Comparing (13), (14), and (15), we obtain

$$
P=\left(\begin{array}{ccccc}
E & H & H & \cdots & H \\
0 & E & H & \cdots & H \\
0 & 0 & E & \cdots & H \\
\vdots & \vdots & \vdots & & \vdots \\
0 & 0 & 0 & \cdots & E
\end{array}\right)
$$

where $E$ and $H$ are $M \times M$ matrices that can be obtained as follows

Let

$$
E=\left(e_{i j}\right), \quad H=\left(h_{i j}\right)
$$

then, for $i, j=0,1, \ldots, M-1$, we have

$$
\begin{aligned}
e_{i j} & =\frac{t_{f}}{2 N} \int_{-1}^{t_{j}} C_{i}(v) d v, \\
h_{i j} & =\frac{t_{f}}{2 N} \int_{-1}^{1} C_{i}(v) d v,
\end{aligned}
$$

where $t_{j}, j=0,1, \ldots, M-1$, are the zeros of the first kind Chebyshev polynomial of order $M$. It is noted that $E$ is the operational matrix of integration for Chebyshev cardinal functions over interval $\left[((n-1) / N) t_{f},(n / N) t_{f}\right)$.

Remark 1. To calculate the entries $e_{i j}$ and $h_{i j}, i, j=0,1$, $\ldots, M-1$, we have

$$
C_{m}(v)=\frac{T_{M}(v)}{T_{M, v}\left(t_{m}\right)\left(v-t_{m}\right)}=\frac{\beta}{T_{M, v}\left(t_{m}\right)} \times \prod_{k=0, k \neq m}^{M-1}\left(v-t_{k}\right),
$$

where $\beta=2^{M-1}$ is the coefficient of $t^{M}$ in the Chebyshev polynomial function $T_{M}(v)$. Using (22) we get

$$
\begin{aligned}
& e_{i j}=\frac{t_{f} \beta}{2 N T_{M, t}\left(t_{i}\right)} \int_{-1}^{t_{j}} \prod_{k=0, k \neq i}^{M-1}\left(v-t_{k}\right) d v, \\
& h_{i j}=\frac{t_{f} \beta}{2 N T_{M, t}\left(t_{i}\right)} \int_{-1}^{1} \prod_{k=0, k \neq i}^{M-1}\left(v-t_{k}\right) d v,
\end{aligned}
$$

for $i, j=0,1, \ldots, M-1$.

2.4. The Operational Matrix of Product. The following property of the product of two hybrid function vectors will also be used. Let

$$
\Theta(t) \Theta^{T}(t) F \simeq \widetilde{F} \Theta(t),
$$

where

$$
F=\left[f_{10}, \ldots, f_{1 M-1}, f_{20}, \ldots, f_{2 M-1}, \ldots, f_{N 0}, \ldots, f_{N M-1}\right]^{T},
$$


and $\widetilde{F}$ is an $M N \times M N$ product operational matrix. To find $\widetilde{F}$, we apply the following procedure. First, by using (11) and (25) we obtain

$$
\Theta(t) \Theta^{T}(t) F=\left(\begin{array}{c}
\theta_{10}(t) \sum_{n=1}^{\infty} \sum_{m=0}^{\infty} f_{n m} \theta_{n m}(t) \\
\theta_{11}(t) \sum_{n=1}^{\infty} \sum_{m=0}^{\infty} f_{n m} \theta_{n m}(t) \\
\vdots \\
\theta_{1 M-1}(t) \sum_{n=1}^{\infty} \sum_{m=0}^{\infty} f_{n m} \theta_{n m}(t) \\
\vdots \\
\theta_{N 0}(t) \sum_{n=1}^{\infty} \sum_{m=0}^{\infty} f_{n m} \theta_{n m}(t) \\
\theta_{N 1}(t) \sum_{n=1}^{\infty} \sum_{m=0}^{\infty} f_{n m} \theta_{n m}(t) \\
\vdots \\
\theta_{N M-1}(t) \sum_{n=1}^{\infty} \sum_{m=0}^{\infty} f_{n m} \theta_{n m}(t)
\end{array}\right) .
$$

Using (9), any function $\theta_{i j}(t) \theta_{l k}(t), i, l=1,2, \ldots, N, j, k=$ $0,1, \ldots, M-1$, can be approximated as

$$
\theta_{i j}(t) \theta_{l k}(t) \simeq \sum_{n=1}^{N} \sum_{m=0}^{M-1} c_{n m} \theta_{n m}(t)
$$

where

$$
\begin{aligned}
c_{n m} & =\theta_{i j}\left(\frac{t_{f}}{2 N}\left(t_{m}+2 n-1\right)\right) \theta_{l k}\left(\frac{t_{f}}{2 N}\left(t_{m}+2 n-1\right)\right) \\
& =\delta_{i n} \delta_{j m} \delta_{l n} \delta_{k m} .
\end{aligned}
$$

So, from (26) and (28), we have

$$
\begin{aligned}
& \Theta(t) \Theta^{T}(t) F \\
& \simeq\left[f_{10} \theta_{10}(t), \ldots, f_{1 M-1} \theta_{1 M-1}(t), \ldots,\right. \\
& \left.\quad f_{N 0} \theta_{N 0}(t), \ldots, f_{N M-1} \theta_{N M-1}(t)\right]^{T} ;
\end{aligned}
$$

therefore, we find the $M N \times M N$ matrix $\widetilde{F}$ as

$$
\begin{aligned}
\widetilde{F}=\operatorname{diag}[ & f_{10}, \ldots, f_{1 M-1}, f_{20}, \ldots, \\
& \left.f_{2 M-1}, \ldots, f_{N 0}, \ldots, f_{N M-1}\right] .
\end{aligned}
$$

Lemma 2. The functions $C_{j}(t), j=0,1, \ldots, M-1$, are orthogonal with respect to $w(t)=1 / \sqrt{1-t^{2}}$ on $[-1,1]$ and satisfy the orthogonality condition

$$
\left\langle C_{i}(t), C_{j}(t)\right\rangle_{w}=\int_{-1}^{1} \frac{C_{i}(t) C_{j}(t)}{\sqrt{1-t^{2}}} d t= \begin{cases}\frac{\pi}{M}, & \text { if } j=i, \\ 0, & \text { if } j \neq i .\end{cases}
$$

Remark 3. Since $\theta_{n, m}(t)$ consists of block-pulse functions and Chebyshev cardinal functions, which are both complete and orthogonal, the set of hybrid of block-pulse functions and Chebyshev cardinal functions is a complete orthogonal set in the Hilbert space $L^{2}\left[0, t_{f}\right)$.

Lemma 4. Let $M N$ vectors $C$ and $C_{p}$ be hybrid functions coefficients of $u(t)$ and $u^{p}(t)$, respectively. If

$$
C=\left[c_{10}, \ldots, c_{1 M-1}, c_{20}, \ldots, c_{2 M-1}, \ldots, c_{N 0}, \ldots, c_{N M-1}\right]^{T},
$$

then

$$
C_{p} \simeq\left[c_{10}^{p}, \ldots, c_{1 M-1}^{p}, c_{20}^{p}, \ldots, c_{2 M-1}^{p}, \ldots, c_{N 0}^{p}, \ldots, c_{N M-1}^{p}\right]^{T},
$$

where $p \geq 1$ is a positive integer.

Proof. When $p=1$, (33) follows at once from $u^{p}(t)=u(t)$. Suppose that (33) holds for $p$; we will deduce it for $p+1$. Since $u^{p+1}(t)=u(t) u^{p}(t)$, from (24) and (30) we have:

$$
\begin{aligned}
u^{p+1}(t) & \simeq\left(C^{T} \Theta(t)\right)\left(C_{p}^{T} \Theta(t)\right) \\
& =C^{T} \Theta(t) \Theta^{T}(t) C_{p} \simeq C^{T} \widetilde{C}_{p} \Theta(t),
\end{aligned}
$$

where $\widetilde{C}_{p}$ can be calculated in a similar way to matrix $\widetilde{F}$ in (24). Now, using (33) we obtain

$$
C_{p+1}=C^{T} \widetilde{C}_{p}=\left[c_{10}^{p+1}, \ldots, c_{1 M-1}^{p+1}, c_{20}^{p+1}, \ldots,\right.
$$

$$
\left.c_{2 M-1}^{p+1}, \ldots, c_{N 0}^{p+1}, \ldots, c_{N M-1}^{p+1}\right]^{T} .
$$

Therefore, (33) holds for $p+1$, and the lemma is established.

\section{Hybrid Functions Method to Solve Duffing-Harmonic Oscillator}

In this section, by using the results obtained in the previous section about hybrid functions, an effective and accurate method for solving Duffing-harmonic oscillator (1) is presented.

Consider the following nonlinear Duffing-harmonic oscillator:

$$
u^{\prime \prime}+\frac{u^{3}}{1+u^{2}}=0, \quad t \in\left[0, t_{f}\right)
$$

with the initial conditions

$$
u(0)=A, \quad u^{\prime}(0)=0 .
$$

At first, we write (36) in the following form:

$$
\left(1+u^{2}\right) u^{\prime \prime}+u^{3}=0 .
$$

Let

$$
u^{\prime \prime}(t) \simeq U^{T} \Theta(t),
$$


TABLE 1: Comparison of various approximate angular frequencies with exact angular frequency.

\begin{tabular}{|c|c|c|c|c|c|c|c|c|}
\hline \multirow{2}{*}{ A } & \multirow{2}{*}{$\omega_{\mathrm{HBM}}$} & \multirow{2}{*}{$\omega_{\mathrm{EBM}}$} & \multirow{2}{*}{$\omega_{\text {Tiw }}$} & \multirow{2}{*}{$\omega_{\mathrm{ex}}$} & \multicolumn{4}{|c|}{ HFM } \\
\hline & & & & & $N$ & $M$ & $t_{f}$ & $\omega_{\mathrm{HFM}}$ \\
\hline 0.01 & 0.00866 & 0.00866 & 0.00866 & 0.00847 & 10 & 5 & 750 & 0.00846 \\
\hline 0.05 & 0.04326 & 0.04326 & 0.04327 & 0.04232 & 10 & 5 & 150 & 0.04236 \\
\hline 0.1 & 0.08628 & 0.08627 & 0.08624 & 0.08439 & 10 & 5 & 75 & 0.08446 \\
\hline 0.5 & 0.39736 & 0.39638 & 0.39423 & 0.38737 & 10 & 5 & 16.5 & 0.38757 \\
\hline 1.0 & 0.65465 & 0.65164 & 0.64359 & 0.63678 & 15 & 5 & 10 & 0.63673 \\
\hline 5.0 & 0.97435 & 0.97343 & 0.96731 & 0.96698 & 14 & 10 & 6.5 & 0.96699 \\
\hline 10.0 & 0.99340 & 0.99314 & 0.99095 & 0.99092 & 14 & 10 & 6.5 & 0.99093 \\
\hline
\end{tabular}

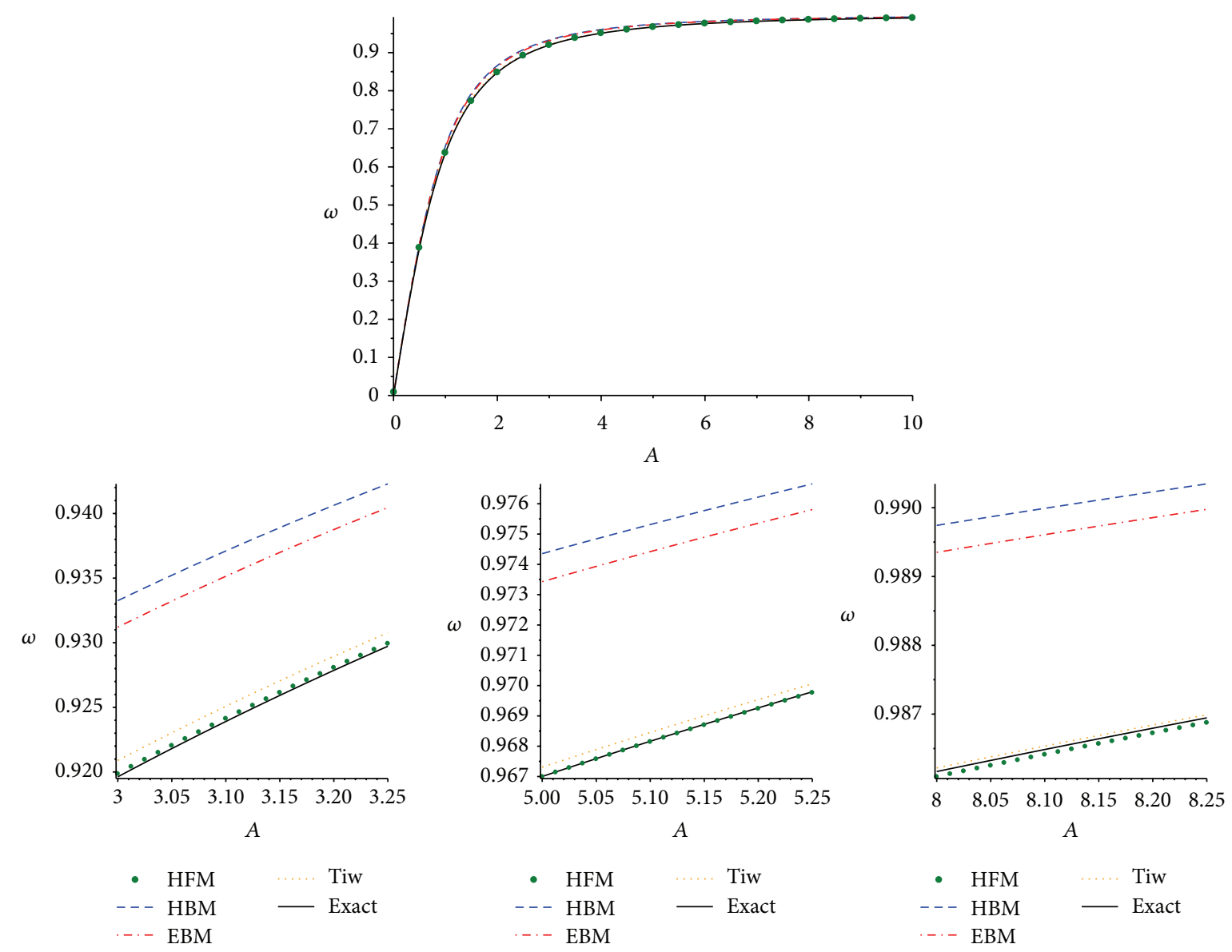

FIGURE 1: Comparison of the approximate frequencies with corresponding exact frequency.

where $\Theta(t)$ is defined in (11) and $U$ is a vector with $M N$ unknowns as follows:

$$
U=\left[u_{10}, \ldots, u_{1 M-1}, u_{20}, \ldots, u_{2 M-1}, \ldots, u_{N 0}, \ldots, u_{N M-1}\right]^{T}
$$

By expanding $u(0)=A$ and $f(t)=1$ in terms of hybrid functions we get

$$
\begin{gathered}
u(0)=A=\mathscr{A}^{T} \Theta(t), \\
f(t)=1=E^{T} \Theta(t),
\end{gathered}
$$

where $\mathscr{A}=[\overbrace{A, A, \ldots, A}^{M N}]^{T}$ and $E=[\overbrace{1,1, \ldots, 1}^{M N}]^{T}$. Integrating (39) from 0 to $t$ and using (41), we obtain

$$
\begin{aligned}
u^{\prime}(t) & =\int_{0}^{t} U^{T} \Theta(s) d s+u^{\prime}(0) \simeq U^{T} P \Theta(t), \\
u(t) & =\int_{0}^{t} u^{\prime}(s) d s+u(0) \\
& \simeq \int_{0}^{t} U^{T} P \Theta(s) d s+\mathscr{A}^{T} \Theta(t) \simeq U^{T} P^{2} \Theta(t)+\mathscr{A}^{T} \Theta(t) \\
& =\mathcal{U}^{T} \Theta(t),
\end{aligned}
$$



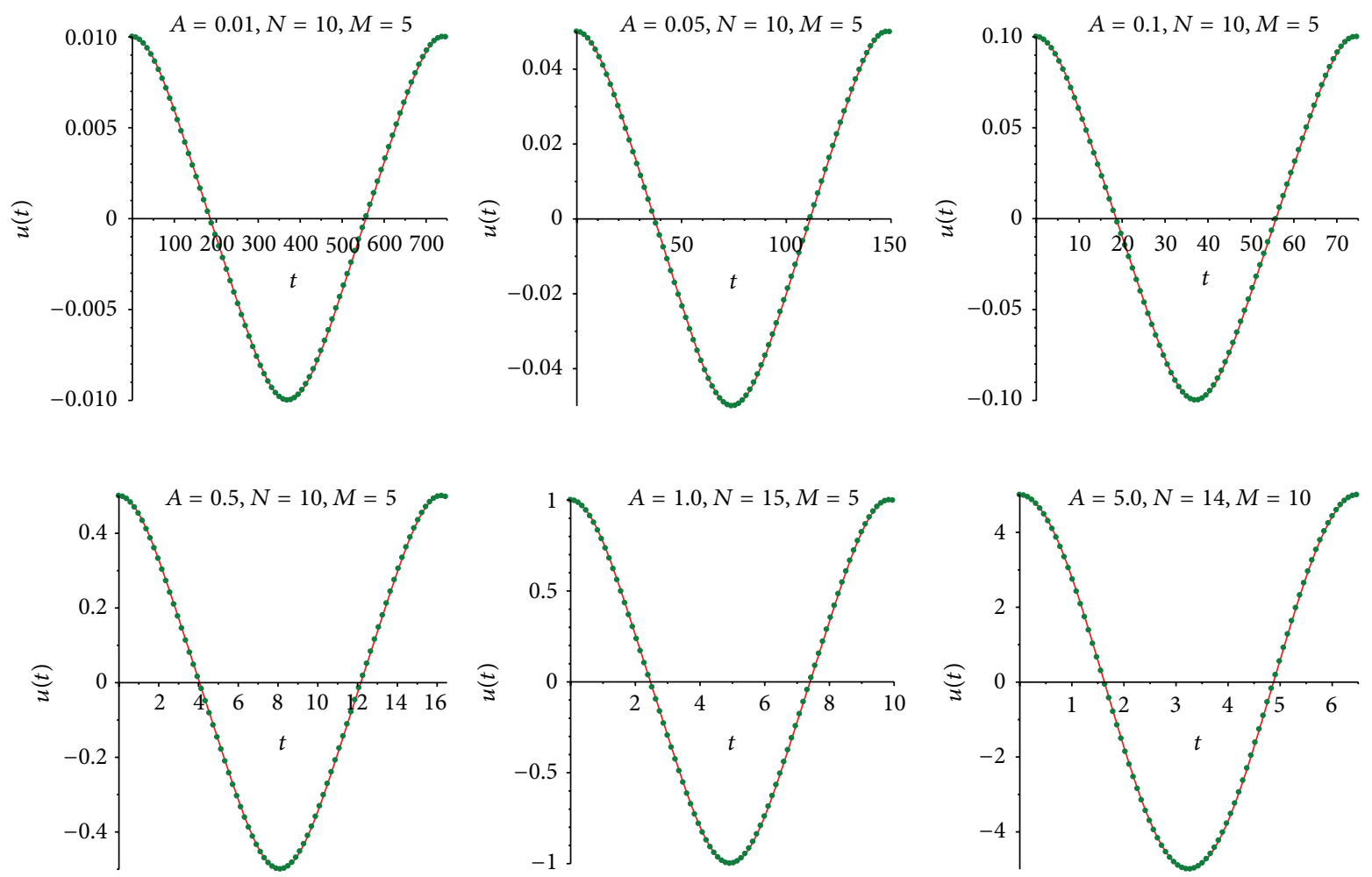

FIGURE 2: Plots of displacement $u$ versus time $t$. Solid line: HFM; solid circle: RK4.

where $\mathcal{U}=\left(U^{T} P^{2}+\mathscr{A}^{T}\right)^{T}$ and $P$ is the operational matrix of integration given in (13). Using Lemma 4 the functions $u^{2}(t)$ and $u^{3}(t)$ can be expanded as

$$
\begin{aligned}
& u^{2}(t) \simeq \mathcal{u}_{2}^{T} \Theta(t), \\
& u^{3}(t) \simeq \mathcal{u}_{3}^{T} \Theta(t) .
\end{aligned}
$$

Therefore, by using (39) and (42)-(45), the right side of (38) can be approximated as

$$
\begin{aligned}
& \left(1+u^{2}(t)\right) u^{\prime \prime}(t)+u^{3}(t) \\
& \simeq\left(E^{T}+\mathcal{U}_{2}^{T}\right) \underbrace{\Theta(t) \Theta^{T}(t) U}_{\widetilde{U} \Theta(t)}+\mathscr{U}_{3}^{T} \Theta(t) \\
& \simeq\left[\left(E^{T}+\mathcal{U}_{2}^{T}\right) \widetilde{U}+\mathscr{U}_{3}^{T}\right] \Theta(t)=0,
\end{aligned}
$$

where $\widetilde{U}$ can be calculated in a similar way to matrix $\widetilde{F}$ in (24). Since the above equation is satisfied for every $t \in\left[0, t_{f}\right)$, we can get

$$
\left(E^{T}+\mathcal{U}_{2}^{T}\right) \widetilde{U}+\mathscr{U}_{3}^{T}=0 .
$$

This is a system of algebraic equations with $M N$ equations and $M N$ unknowns, which can be solved by Newton's iteration method to obtain the unknown vector $U$.
TABLE 2: Error percentage comparison between previous results and HFM with various $A$.

\begin{tabular}{lcccc}
\hline \multirow{2}{*}{$A$} & \multicolumn{4}{c}{ Error percentage } \\
& HBM & EBM & Tiw & HFM \\
\hline 0.01 & 2.242 & 2.242 & 2.242 & 0.118 \\
0.05 & 2.223 & 2.219 & 2.212 & 0.095 \\
0.1 & 2.239 & 2.225 & 2.197 & 0.083 \\
0.5 & 2.579 & 2.326 & 1.771 & 0.052 \\
1.0 & 2.807 & 2.334 & 1.070 & 0.008 \\
5.0 & 0.763 & 0.667 & 0.034 & 0.001 \\
10.0 & 0.250 & 0.224 & 0.003 & 0.001 \\
\hline
\end{tabular}

Remark 5. The approximate period and frequency of the hybrid functions method (HFM) can be obtained as follows:

$$
T_{\mathrm{HFM}}=\alpha, \quad \omega_{\mathrm{HFM}}=\frac{2 \pi}{\alpha},
$$

where $\alpha$ is the first positive root of equation $u(t)-A=0$. Here, we use the famous Newton's iteration method for finding a proper approximation $\alpha$ of nonlinear equation $u(t)-A=0$, in the following form:

$$
\alpha_{n+1}=\alpha_{n}-\frac{F\left(\alpha_{n}\right)}{F^{\prime}\left(\alpha_{n}\right)}, \quad n=0,1, \ldots,
$$

where $F(t)=u(t)-A=\left(\mathscr{U}^{T}-\mathscr{A}^{T}\right) \Theta(t)$ and $\alpha_{0}$ is initial approximation. 

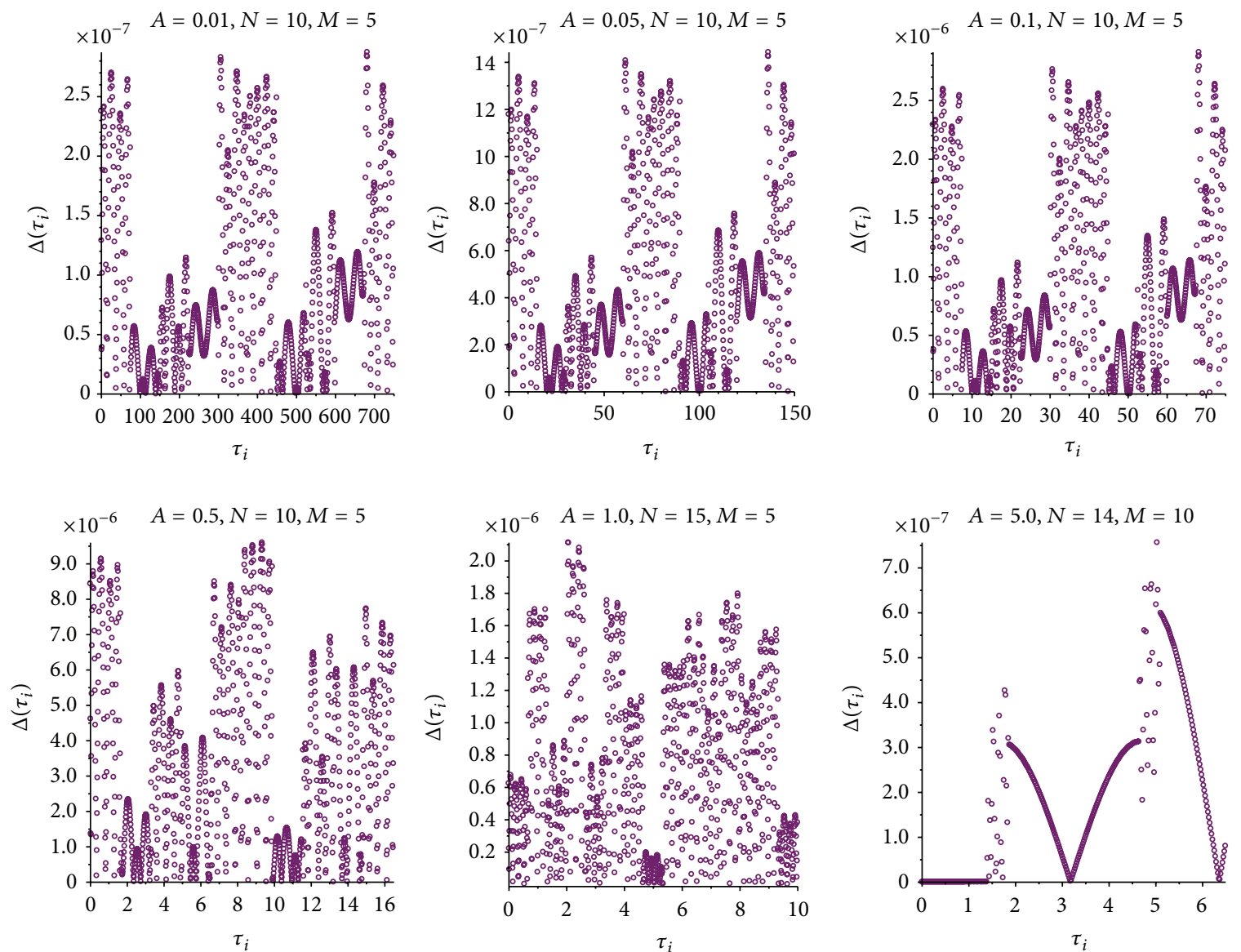

FIGURE 3: Plots of error values $\Delta\left(r_{i}\right)$ with $\beta=50$.

\section{Results and Discussions}

In this section, we illustrate the accuracy of the hybrid functions method (HFM) by comparing the approximate solutions previously obtained with the exact angular frequency $\omega_{\text {ex }}$. All the results obtained here are computed using the Intel Pentium 5, 2.2 GHz processor and using Maple 17 with 64-digit precision.

The exact angular frequency, $\omega_{\mathrm{ex}}$, of the Duffingharmonic oscillator was found by Lim and $\mathrm{Wu}$ in [32] as

$$
\omega_{\mathrm{ex}}=\frac{\pi}{2}\left(\int_{0}^{\pi / 2} \frac{A \cos t d t}{\sqrt{A^{2} \cos ^{2} t+\ln \left[1-A^{2} \cos ^{2} t /\left(1+A^{2}\right)\right]}}\right)^{-1} .
$$

By using alternative form (38) and applying the harmonic balance method (HBM) [13], Mickens [31] obtained the first approximate angular frequency

$$
\omega_{\mathrm{HBM}}=\left(\frac{3 A^{2}}{4+3 A^{2}}\right)^{1 / 2}
$$

Ozis and Yildirim [35] obtained the angular frequency using the energy balance method (EBM) in the following form:

$$
\omega_{\mathrm{EBM}}=\left(1-\frac{2}{A^{2}} \ln \left(\frac{1+A^{2}}{1+\left(A^{2} / 2\right)}\right)\right)^{1 / 2} .
$$

Ganji et al. in [34] obtained the same approximation as that in (52). Using a single-term approximate solution $u(t)=$ $A \cos (\omega t)$ to (36) and the Ritz procedure [40], Tiwari et al. [41] obtained an approximate angular frequency as follows:

$$
\omega_{\text {Tiw }}=\left(1+\frac{2}{A^{2}}\left(\frac{1}{\sqrt{1+A^{2}}}-1\right)\right)^{1 / 2} \text {. }
$$

The computed results for the HFM frequency $\omega_{\mathrm{HFM}}$ with exact frequency $\omega_{\text {ex }}[32], \mathrm{HBM}$ frequency $\omega_{\mathrm{HBM}}$ [31], EBM frequency $\omega_{\mathrm{EBM}}[35]$, and Tiwari's frequency $\omega_{\text {Tiw }}[41]$ are listed in Tables 1 and 2. Table 2 shows that the maximum percentage error between $\omega_{\text {HFM }}$ and exact frequency $\omega_{\text {ex }}$ is $0.118 \%$. Comparison of the exact frequency $\omega_{\text {ex }}$ obtained by (50) with $\omega_{\mathrm{HBM}}, \omega_{\mathrm{EBM}}, \omega_{\mathrm{Tiw}}$, and $\omega_{\mathrm{HFM}}$ is shown in Figure 1 for $0 \leq A \leq 10,3.0 \leq A \leq 3.25,5.0 \leq A \leq 5.25$, and $8.0 \leq A \leq 8.25$. 
From Figure 1 and Tables 1 and 2, it can be observed that the hybrid functions method (HFM) yields excellent approximate frequencies for both small and large amplitudes.

Figures 2 and 3 give a comparison between the present HFM results and the numerical results obtained by using the 4 th order Runge-Kutta method (RK4) with time step $\Delta t=$ 0.001 , where

$$
\Delta\left(\tau_{i}\right)=\left|u_{\mathrm{HFM}}\left(\tau_{i}\right)-u_{\mathrm{RK} 4}\left(\tau_{i}\right)\right|, \quad i=0,1, \ldots, \frac{t_{f}}{\beta \Delta t},
$$

$\tau_{i}=i \beta \Delta t, i=0,1, \ldots, t_{f} /(\beta \Delta t)$, and $\beta \in \mathbb{N}$. It can be seen from these figures that the solutions obtained by the proposed procedure are in good agreement with the RK4 based solutions.

\section{Conclusion}

In this paper, we presented a numerical scheme based on hybrid block-pulse functions and Chebyshev cardinal functions for solving Duffing-harmonic oscillator. This algorithm reduces the solution of Duffing-harmonic oscillator differential equation to the solution of a system of algebraic equations in matrix form. The merit of this method is that the system of equations obtained for the solution does not need to consider collocation points; this means that the system of equations is obtained directly. A comparative study between HBM [31], EBM [35], Tiwari's method [41], and the proposed method was discussed in Section 4. The obtained results showed that the HFM is accurate, capable, and effective technique for the solution of the Duffing-harmonic oscillator. Further research can concentrate on other strongly nonlinear oscillators and more complicated cases.

\section{Conflict of Interests}

The authors declare that there is no conflict of interests regarding the publication of this paper.

\section{Acknowledgment}

The authors are very grateful to both reviewers for carefully reading the paper and for their comments and suggestions which have improved the paper.

\section{References}

[1] A. H. Nayfeh, Perturbation Methods, John Wiley \& Sons, New York, NY, USA, 1973.

[2] A. H. Nayfeh and D. T. Mook, Nonlinear Oscillations, John Wiley, New York, NY, USA, 1979.

[3] N. Krylov and N. Bogolioubov, Introduction to Nonlinear Mechanics, Princeton University Press, Princeton, NJ, USA, 1943.

[4] N. N. Bogolioubov and Y. A. Mitropolsky, Asymptotic Methods in the Theory of Nonlinear Oscillations, Gordon and Breach, New York, NY, USA, 1961.

[5] J. He, "Modified Lindstedt-Poincare methods for some strongly non-linear oscillations: part II: a new transformation," International Journal of Non-Linear Mechanics, vol. 37, no. 2, pp. 315$320,2002$.
[6] J. H. He, "Modified straightforward expansion," Meccanica, vol. 34, no. 4, pp. 287-289, 1999.

[7] J. He, "Preliminary report on the energy balance for nonlinear oscillations," Mechanics Research Communications, vol. 29, no. 2-3, pp. 107-111, 2002.

[8] J. H. He, "Iteration perturbation method for strongly nonlinear oscillations," Journal of Vibration and Control, vol. 7, no. 5, pp. 631-642, 2001.

[9] J. H. He, "Bookkeeping parameter in perturbation methods," International Journal of Nonlinear Sciences and Numerical Simulation, vol. 2, no. 3, pp. 257-264, 2001.

[10] J. He, "Some asymptotic methods for strongly nonlinear equations," International Journal of Modern Physics B, vol. 20, no. 10, pp. 1141-1199, 2006.

[11] J. He, "Max-min approach to nonlinear oscillators," International Journal of Nonlinear Sciences and Numerical Simulation, vol. 9, no. 2, pp. 207-210, 2008.

[12] R. E. Mickens, "Iteration procedure for determining approximate solutions to nonlinear oscillator equations," Journal of Sound and Vibration, vol. 116, no. 1, pp. 185-187, 1987.

[13] R. E. Mickens, Oscillations in Planar Dynamics Systems, World Scientific, Singapore, 1996.

[14] G. Adomian, Solving Frontier Problems of Physics: The Composition Method, Kluwer Academic Publishers, Boston, Mass, USA, 1994.

[15] J. He, "Variational iteration method-a kind of non-linear analytical technique: some examples," International Journal of Non-Linear Mechanics, vol. 34, no. 4, pp. 699-708, 1999.

[16] M. Heydari, S. M. Hosseini, G. B. Loghmani, and D. D. Ganji, "Solution of strongly nonlinear oscillators using modified variational iteration method," International Journal of Nonlinear Dynamics in Engineering and Sciences, vol. 3, pp. 33-45, 2011.

[17] M. Heydari, G. B. Loghmani, and S. M. Hosseini, "An improved piecewise variational iteration method for solving strongly nonlinear oscillators," Computational and Applied Mathematics, 2014.

[18] J. He, "Homotopy perturbation technique," Computer Methods in Applied Mechanics and Engineering, vol. 178, no. 3-4, pp. 257262, 1999.

[19] S. Momani and V. S. Ertürk, "Solutions of non-linear oscillators by the modified differential transform method," Computers \& Mathematics with Applications, vol. 55, no. 4, pp. 833-842, 2008.

[20] S. Momani, G. H. Erjaee, and M. H. Alnasr, "The modified homotopy perturbation method for solving strongly nonlinear oscillators," Computers \& Mathematics with Applications, vol. 58, no. 11-12, pp. 2209-2220, 2009.

[21] M. Razzaghi, J. Nazarzadeh, and K. Y. Nikravesh, “A collocation method for optimal control of linear systems with inequality constraints," Mathematical Problems in Engineering, vol. 3, no. 6, pp. 503-515, 1998.

[22] M. Razzaghi and H. Marzban, "Direct method for variational problems via hybrid of block-pulse and Chebyshev functions," Mathematical Problems in Engineering, vol. 6, no. 1, pp. 85-97, 2000.

[23] M. Razzaghi and H. R. Marzban, "A hybrid domain analysis for systems with delays in state and control," Mathematical Problems in Engineering. Theory, Methods and Applications, vol. 7, no. 4, pp. 337-353, 2001.

[24] H. R. Marzban and M. Razzaghi, "Solution of time-varying delay systems by hybrid functions," Mathematics and Computers in Simulation, vol. 64, no. 6, pp. 597-607, 2004. 
[25] K. Maleknejad and M. Tavassoli Kajani, "Solving linear integrodifferential equation system by Galerkin methods with hydrid functions," Applied Mathematics and Computation, vol. 159, no. 3, pp. 603-612, 2004.

[26] H. R. Marzban and M. Razzaghi, "Solution of multi-delay systems using hybrid of block-pulse functions and Taylor series," Journal of Sound and Vibration, vol. 292, no. 3-5, pp. 954-963, 2006.

[27] H. R. Marzban, S. M. Hoseini, and M. Razzaghi, "Solution of Volterra's population model via block-pulse functions and Lagrange-interpolating polynomials," Mathematical Methods in the Applied Sciences, vol. 32, no. 2, pp. 127-134, 2009.

[28] H. R. Marzban, H. R. Tabrizidooz, and M. Razzaghi, "Hybrid functions for nonlinear initial-value problems with applications to Lane-Emden type equations," Physics Letters A, vol. 372, no. 37, pp. 5883-5886, 2008.

[29] M. Razzaghi, "Optimization of time delay systems by hybrid functions," Optimization and Engineering, vol. 10, no. 3, pp. 363376, 2009.

[30] H. R. Marzban, H. R. Tabrizidooz, and M. Razzaghi, "A composite collocation method for the nonlinear mixed VolterraFredholm-Hammerstein integral equations," Communications in Nonlinear Science and Numerical Simulation, vol. 16, no. 3, pp. 1186-1194, 2011.

[31] R. E. Mickens, "Mathematical and numerical study of the Duffing-harmonic oscillator," Journal of Sound and Vibration, vol. 244, no. 3, pp. 563-567, 2001.

[32] C. W. Lim and B. S. Wu, "A new analytical approach to the Duffing-harmonic oscillator," Physics Letters A, vol. 311, no. 45, pp. 365-373, 2003.

[33] C. W. Lim, B. S. Wu, and W. P. Sun, "Higher accuracy analytical approximations to the Duffing-harmonic oscillator," Journal of Sound and Vibration, vol. 296, no. 4-5, pp. 1039-1045, 2006.

[34] S. S. Ganji, D. D. Ganji, Z. Z. Ganji, and S. Karimpour, "Periodic solution for strongly nonlinear vibration systems by He's energy balance method," Acta Applicandae Mathematicae, vol. 106, no. 1, pp. 79-92, 2009.

[35] T. Ozis and A. Yildirim, "Determination of the frequencyamplitude relation for a Duffing-harmonic oscillator by the energy balance method," Computers \& Mathematics with Applications, vol. 54, no. 7-8, pp. 1184-1187, 2007.

[36] J. P. Boyd, Chebyshev and Fourier Spectral Methods, Dover Publications, 2000.

[37] M. Heydari, Z. Avazzadeh, and G. B. Loghmani, "Chebyshev cardinal functions for solving Volterra-Fredholm integrodifferential equations using operational matrices," Iranian Journal of Science and Technology A, vol. 36, no. 1, pp. 13-24, 2012.

[38] M. Heydari, G. B. Loghmani, and S. M. Hosseini, "Operational matrices of Chebyshev cardinal functions and their application for solving delay differential equations arising in electrodynamics with error estimation," Applied Mathematical Modelling, vol. 37, no. 14-15, pp. 7789-7809, 2013.

[39] J. C. Mason and D. C. Handscomb, Chebyshev Polynomials, CRC Press, Boca Raton, Fla, USA, 2003.

[40] H. N. Abramson, "Nonlinear vibration," in Shock and Vibration Handbook, C. M. Harris, Ed., McGraw-Hill, New York, NY, USA, 1988.

[41] S. B. Tiwari, B. Nageswara Rao, N. Shivakumar Swamy, K. S. Sai, and H. R. Nataraja, "Analytical study on a Duffing-harmonic oscillator," Journal of Sound and Vibration, vol. 285, no. 4-5, pp. 1217-1222, 2005. 


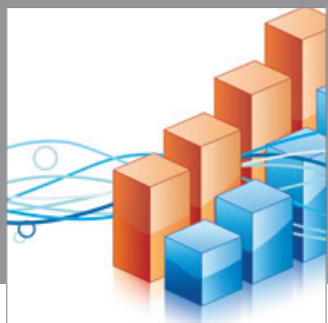

Advances in

Operations Research

mansans

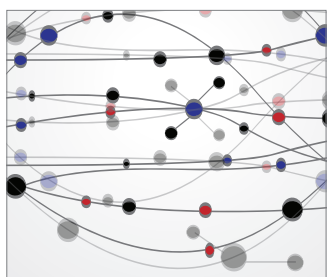

The Scientific World Journal
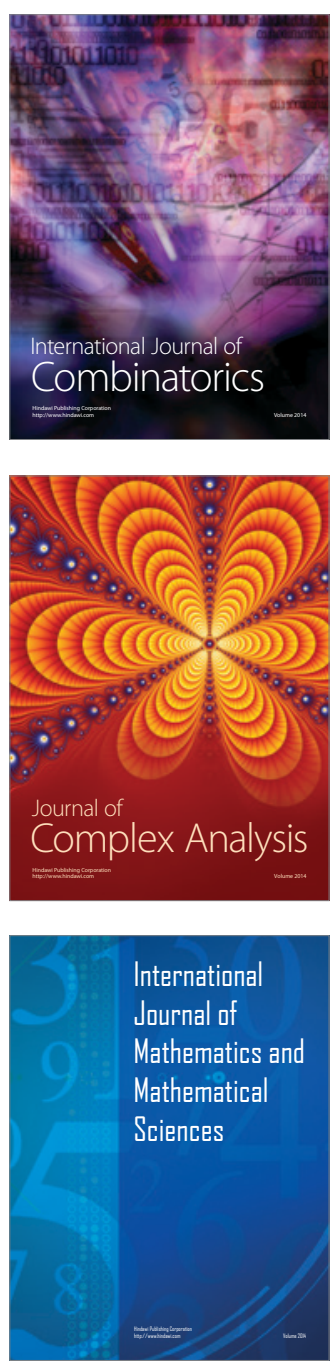
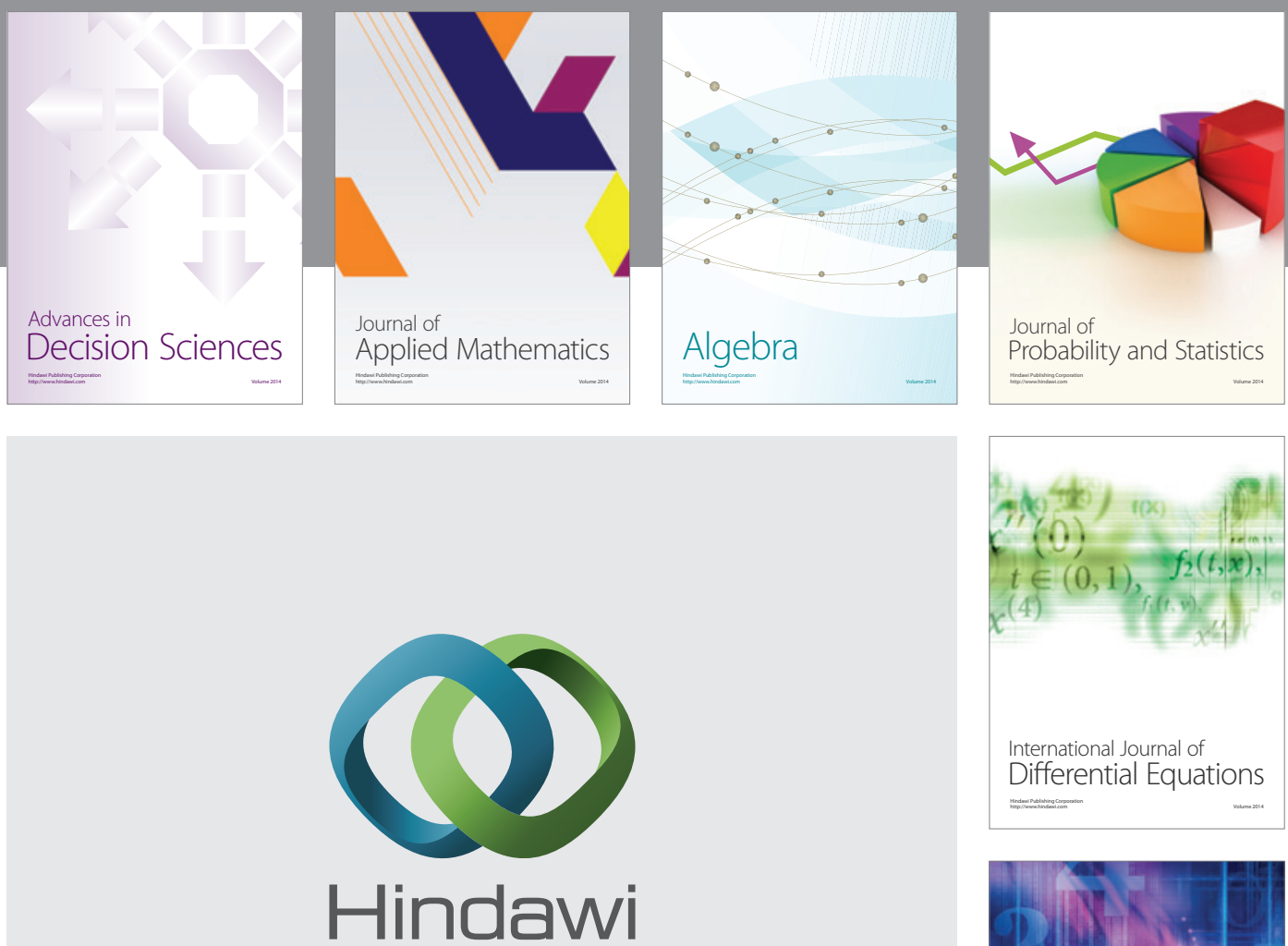

Submit your manuscripts at http://www.hindawi.com
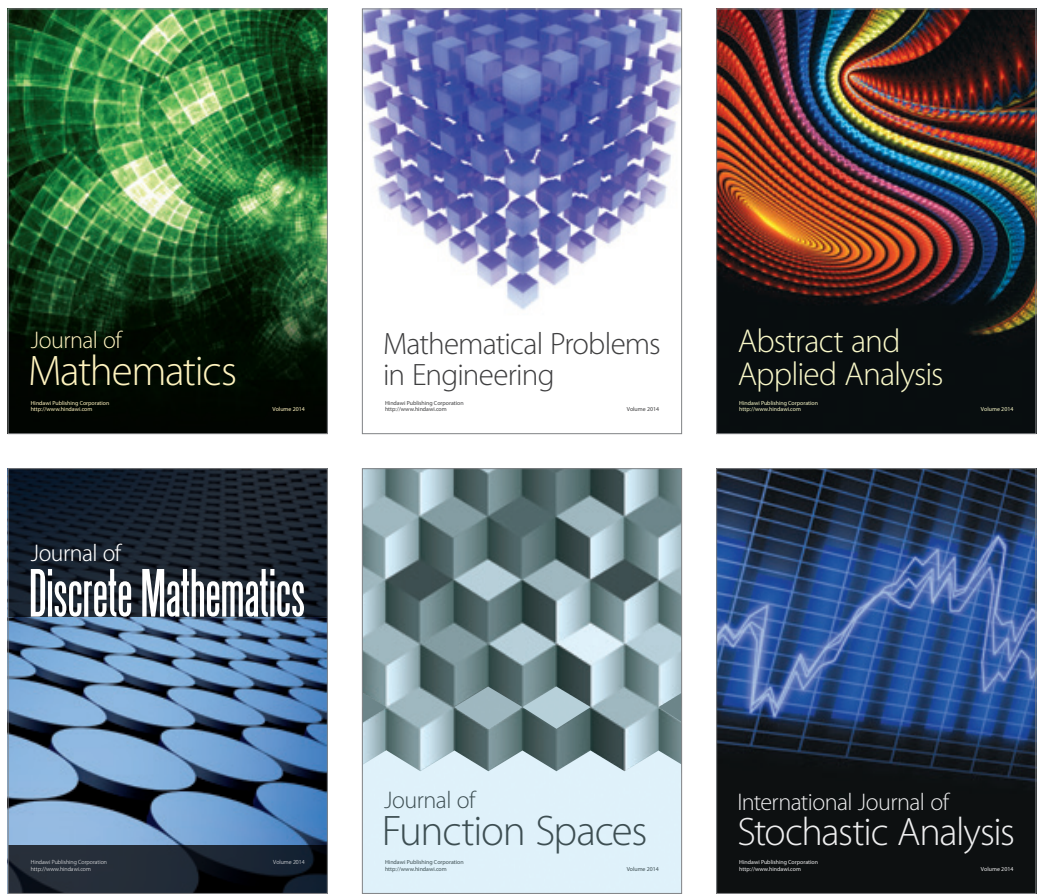

Journal of

Function Spaces

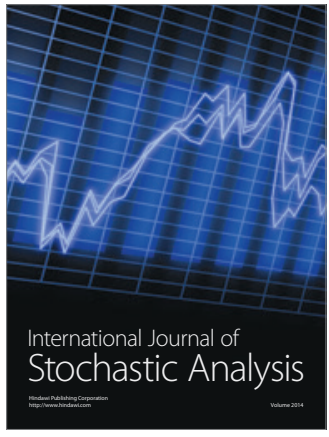

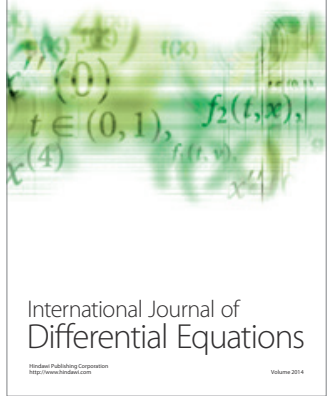
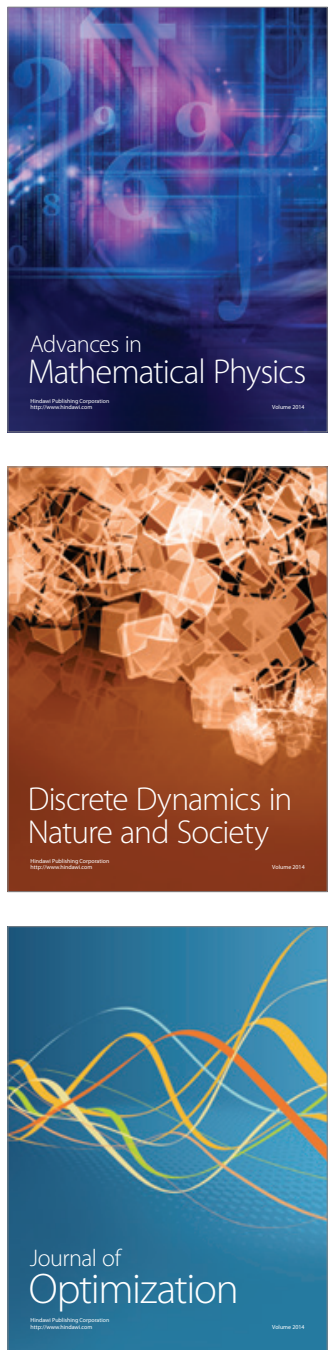